\title{
Microwave-assisted synthesis of fluorinated coumarino sulfonamides
}

\author{
Hua Zuo, ${ }^{\text {a,c }}$ Geo Jose, ${ }^{\text {b }}$ Zu-bo Li, ${ }^{\mathrm{c}}$ Bu-Hyun Moon, ${ }^{\mathrm{d}}$ Dong-Soo Shin, ${ }^{\mathrm{a}^{*}}$ \\ and Manjunath Ghate ${ }^{b^{*}}$ \\ ${ }^{a}$ Department of Chemistry, Changwon National University, Changwon, Korea, 641-773 \\ ${ }^{b}$ Department of Pharmaceutical Chemistry, Krupanidhi College of Pharmacy, Koramangala \\ Bangalore, India, 110034 \\ ${ }^{c}$ College of Pharmacy, Southwest University, Chongqing, China, 400715 \\ ${ }^{d}$ Hwasung Chemical Co., Ulsan, S. Korea, 689-871 \\ E-mail:dsshin@changwon.ac.kr,ghate72@yahoo.com
}

\begin{abstract}
An efficient microwave-assisted protocol for the synthesis of fluorinated coumarino sulfonamides $\mathbf{3}$ was achieved. First the fluoro arylamines were reacted with methyl 2chlorosulfonyl acetate to afford compounds $\mathbf{2}$ followed by Knoevenagel condensation with substituted salicylaldehydes to give fluorinated coumarino sulfonamides 3. Comparison of conventional and microwave irradiation showed the later procedure required shorter reaction times, generally gave higher yields and was applicable to a larger set of substrates.
\end{abstract}

Keywords: Coumarin, fluorinated, sulfonamide, microwave irradiation, Knoevenagel condensation

\section{Introduction}

Coumarin and its derivatives occur widely in nature, and have been exploited in biological, chemical and physical fields. They are one of the most important classes of organic compounds and they are found to possess several biological activities. ${ }^{1}$ Conventional routes for the synthesis of coumarin-containing compounds involve the Pechmann reaction - condensation of phenols and $\beta$-keto esters - in some cases reaction mixtures were allowed to stand overnight or for a number of days or were heated above $150{ }^{\circ} \mathrm{C}$. Chromones may also be formed as byproducts under some conditions. ${ }^{2}$ Microwave-assisted enhancement of Pechmann reactions was recently shown to have some advantages but the yields of coumarins thus obtained were very low. ${ }^{3}$ Amongst new catalysts, are ionic liquid $[\mathrm{bmim}]\left[\mathrm{HSO}_{4}\right]$ reported by Singh et al., ${ }^{4}$ a polyaniline supported acid catalyst developed by Palaniappan, ${ }^{5}$ and Nafion resin/silica nanocomposites 
desribed by Hölderich ${ }^{6}$ were all used to catalyze Pechmann reactions. However, complicated procedures were required for the preparation of the catalysts at high temperature.

Recently, a new method involving $o$-iodophenols reacting with terminal alkynes under $\mathrm{CO}$ gas with $\mathrm{Pd}(\mathrm{OAc})_{2}$ in the presence of pyridine generated coumarins ${ }^{7}$ but many expensive starting materials were used and the procedure was very complicated. Other methods such as Knoevenagel condensation of salicylaldehydes with malonic acid, malonic esters, cyanoacetic esters or Meldrum's acid, ${ }^{8,9}$ Perkin reaction, ${ }^{10}$ and Wittig reaction in $N, N$-diethylaniline ${ }^{11}$ were also applied for the synthesis of coumarins. However, all reported methods have disadvantages (harsh reaction conditions, low yields, difficult purification or expensive catalysts and complicated procedure for the preparation of catalysts), making the development of a new reliable high-yielding method for the synthesis of coumarins desirable.

With the rapid use of microwave activation as a non-conventional energy source in organic chemistry, numerous organic reactions such as acylation and alkylation reactions, aromatic and nucleophilic substitutions, condensations, cycloadditions, protection and deprotection reactions, esterifications and transesterifications, heterocyclizations, rearrangements, organometallic reactions, oxidations and reductions assisted by microwave heating have been widely desribed. ${ }^{12}$ We report here a rapid and efficient microwave-accelerated method to synthesize fluorinated coumarino sulfonamides by Knoevenagel condensation of methyl fluoro anilino sulfonylacetates with substituted salicylaldehydes. Our aim was to introduce fluoro and sulfonamide moieties into coumarin side-chains hoping for an improvement of biological activity because incorporation of fluorine to various heterocycles is known to influence the biological/pharmacological activity. Further, the sulfonamide moiety itself possesses important antibacterial, ${ }^{13}$ anti-inflammatory, ${ }^{14}$ and antitumor activity. ${ }^{15}$

\section{Results and Discussion}

All the reactions were carried out under both microwave irradiation and normal reflux conditions. The title compounds, fluorinated coumarino sulfonamides 3 were prepared via a twostep reaction. The first step involves reaction of fluoro arylamines $\mathbf{1}$ and methyl 2-chlorosulfonyl acetate to give 2. The second step involves Knoevenagel condensation of anilino sulfonylacetates $\mathbf{2}$ with substituted salicylaldehydes to afford fluorinated coumarino sulfonamides $\mathbf{3}$ as shown in Scheme 1. Comparisons of the two steps by conventional and microwave methods are depicted in Table 1. Formation of the desired compounds was accelerated by microwave irradiation being obtained in 5-12 minutes with higher yields as compared with the conventional method. 
Table 1. Synthesis of coumarin-3-sulfonamides 3a-3r by microwave irradiation and conventional heating

\begin{tabular}{|c|c|c|c|c|c|c|c|c|c|c|}
\hline \multirow[b]{2}{*}{ Entry } & \multirow[b]{2}{*}{$\mathrm{R}_{1}$} & \multirow[b]{2}{*}{$\mathrm{R}_{2}$} & \multicolumn{2}{|c|}{ Reaction time $^{a}$} & \multicolumn{2}{|c|}{ Yield ${ }^{\mathrm{a}}(\%)$} & \multicolumn{2}{|c|}{ Reaction time $^{\mathrm{b}}$} & \multicolumn{2}{|c|}{ Yield $^{\mathrm{b}}(\%)$} \\
\hline & & & $\begin{array}{c}\text { Traditional } \\
\text { heating } \\
\text { (h) }\end{array}$ & $\begin{array}{l}\text { Microwave } \\
\text { Irradiation } \\
\quad(\mathbf{m i n})\end{array}$ & $\begin{array}{c}\text { Traditional } \\
\text { heating }\end{array}$ & $\begin{array}{l}\text { Microwave } \\
\text { irradiation }\end{array}$ & $\begin{array}{l}\text { Traditional } \\
\text { heating } \\
\text { (h) }\end{array}$ & $\begin{array}{c}\text { Microwave } \\
\text { irradiation } \\
(\mathbf{m i n})\end{array}$ & $\begin{array}{l}\text { Traditional } \\
\text { heating }\end{array}$ & $\begin{array}{l}\text { Microwave } \\
\text { irradiation }\end{array}$ \\
\hline $3 a$ & $2-\mathrm{F}$ & $\mathrm{H}$ & 8 & 5 & 78 & 84 & 3 & 4 & 80 & 86 \\
\hline $\mathbf{3 b}$ & $4-\mathrm{F}$ & $\mathrm{H}$ & 8 & 5 & 80 & 86 & 3 & 4 & 82 & 87 \\
\hline $3 c$ & $3-\mathrm{Cl}, 4-\mathrm{F}$ & $\mathrm{H}$ & 8 & 4 & 71 & 76 & 3 & 4 & 72 & 82 \\
\hline 3d & $2-\mathrm{F}$ & $6-\mathrm{Cl}$ & 8 & 8 & 78 & 85 & 3 & 4 & 82 & 86 \\
\hline $3 e$ & $4-\mathrm{F}$ & $6-\mathrm{Cl}$ & 8 & 8 & 78 & 86 & 3 & 4 & 78 & 84 \\
\hline $3 f$ & $3-\mathrm{Cl}, 4-\mathrm{F}$ & $6-\mathrm{Cl}$ & 8 & 8 & 84 & 88 & 3 & 4 & 80 & 93 \\
\hline $3 g$ & $2-\mathrm{F}$ & $6-\mathrm{Br}$ & 8 & 10 & 82 & 84 & 3 & 4 & 82 & 84 \\
\hline $3 h$ & $4-\mathrm{F}$ & $6-\mathrm{Br}$ & 10 & 8 & 80 & 86 & 3 & 5 & 74 & 78 \\
\hline $3 \mathbf{i}$ & $3-\mathrm{Cl}, 4-\mathrm{F}$ & $6-\mathrm{Br}$ & 10 & 10 & 78 & 85 & 3 & 5 & 72 & 74 \\
\hline $3 \mathbf{j}$ & $2-\mathrm{F}$ & $\begin{array}{c}8- \\
\mathrm{OCH}_{3}\end{array}$ & 10 & 10 & 85 & 92 & 3 & 5 & 80 & 84 \\
\hline $3 k$ & $4-\mathrm{F}$ & $\begin{array}{c}8- \\
\mathrm{OCH}_{3}\end{array}$ & 10 & 10 & 86 & 90 & 3 & 5 & 72 & 76 \\
\hline 31 & $3-\mathrm{Cl}, 4-\mathrm{F}$ & $\begin{array}{c}8- \\
\mathrm{OCH}_{3}\end{array}$ & 10 & 8 & 82 & 86 & 3 & 5 & 70 & 86 \\
\hline $3 m$ & $2-F$ & $6-\mathrm{CH}_{3}$ & 10 & 12 & 70 & 74 & 3 & 5 & 80 & 92 \\
\hline $3 n$ & $4-\mathrm{F}$ & 6- $\mathrm{CH}_{3}$ & 10 & 12 & 79 & 84 & 3 & 5 & 72 & 80 \\
\hline 30 & $3-\mathrm{Cl}, 4-\mathrm{F}$ & $6-\mathrm{CH}_{3}$ & 10 & 12 & 77 & 80 & 3 & 5 & 80 & 86 \\
\hline $3 p$ & $2-\mathrm{F}$ & $6-\mathrm{NO}_{2}$ & 10 & 12 & 78 & 82 & 3 & 5 & 78 & 86 \\
\hline $3 q$ & $4-\mathrm{F}$ & $6-\mathrm{NO}_{2}$ & 10 & 12 & 71 & 80 & 3 & 5 & 70 & 76 \\
\hline $3 r$ & 3-Cl, 4-F & $6-\mathrm{NO}_{2}$ & 10 & 12 & 72 & 78 & 3 & 5 & 82 & 88 \\
\hline
\end{tabular}

${ }^{\mathrm{a}}$ Reaction time and isolated yields in step $1 .{ }^{\mathrm{b}}$ Reaction time and isolated yields in step 2. 

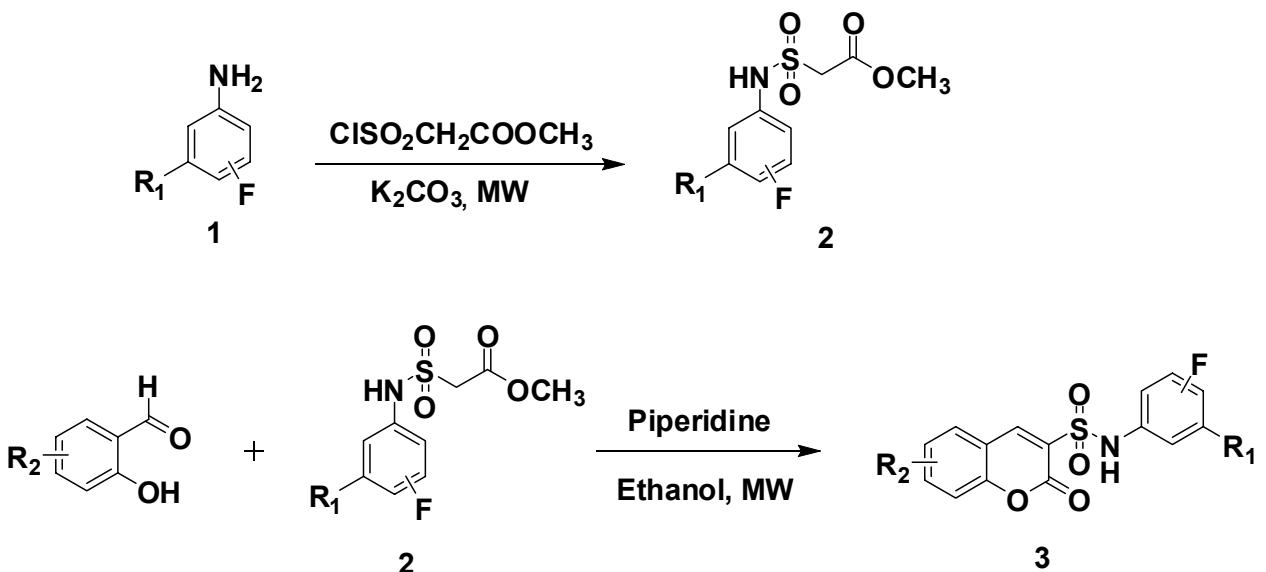

Scheme 1. Synthesis of fluorinated coumarino sulfonamide derivatives 3a-3r.

In a typical experiment, fluoro arylamines $\mathbf{1}$ and the sulfonylchloride were mixed in a sealed tube to afford compounds $\mathbf{2}$. The Knoevenagel condensation of $\mathbf{2}$ with substituted salicylaldehydes led to $N$-fluoroaryl-coumarin-3-sulfonamides 3 (Scheme 1). The different entries and isolated yields in comparison with those in conventional condition are given in Table 1. The reactions were carried out using three different fluoro arylamines $\mathbf{1}$ and six substituted salicylaldehydes.

This process worked well for all types of fluoro aromatic amines and salicylaldehydes, regardless of their electronic and steric nature, giving the desired products in a much shorter time and with better yields as compared to those from conventional conditions.

\section{Conclusions}

To summarise, we have developed an efficient microwave-assisted protocol for the synthesis of fluorinated coumarino sulfonamides starting with fluoro arylamines and methyl 2-chlorosulfonyl acetate followed by Knoevenagel condensation of the obtained anilino sulfonylacetates with substituted salicylaldehydes to afford fluorinated coumarino sulfonamides. Compared with the conventional method, this procedure uses more friendly reaction conditions, shorter reaction times, generally gives higher yields and has been applied to a larger set of substrates. Most of these new compounds exhibit good biological activities. Further, bioassays are underway.

\section{Experimental Section}

General Procedures. Melting points were measured on a Buchi instrument using open capillaries uncorrected. IR spectra were recorded with an IR spectrophotometer Nicolet impact spectrometer using $\mathrm{KBr}$ pellets and ${ }^{1} \mathrm{H}$ NMR spectra were recorded on Bruker $300 \mathrm{MHz}$ 
spectrometer in $\mathrm{CDCl}_{3}+\mathrm{DMSO}-d_{6}$ with TMS as internal reference. The chemical shifts are expressed as $\delta \mathrm{ppm}$ and coupling constants are in $\mathrm{Hz}$. All new compounds were analyzed for $\mathrm{C}$, $\mathrm{H}, \mathrm{N}$ and the results were in acceptable range. Microwave $\mathrm{KMIC}-1.5 \mathrm{KW}$ (domestic oven made in Korean Microwave Instrument Co.) was used to carry out the reactions.

\section{General procedure for the microwave-assisted synthesis of title compounds 3}

Reactions were carried out in a sealed pressure tube in a microwave oven. The tube was irradiated for 1 minute and then the oven was opened, and the hot water contained in the beaker was changed. After 1 minute resting time, another 1 minute of irradiation was applied followed by 1 minute of resting and water changed repeatedly until the reaction was completed. When a microwave-active solvent ethanol and few drops piperidine was used, the sample was irradiated with 30 seconds of resting and the water was changed every time. To ensure that the temperature of the solvent did not rise too much, the volume of water used was at least 3-4 times that of the organic solvent. The reaction was monitored by checking the TLC every minute in the first case. All the other reactions were carried out by checking the TLC after 5 minutes.

$\boldsymbol{N}$-(2-Fluorophenyl)-coumarin-3-sulfonamide (3a). Mp 204-206 ${ }^{\circ} \mathrm{C}$; ${ }^{1} \mathrm{H}$ NMR (300 MHz, $\left.\mathrm{CDCl}_{3}+\mathrm{DMSO}_{\mathrm{d}}\right): \delta$ 6.40-7.60 (m, 6H), $7.61(\mathrm{~d}, J=8.2 \mathrm{~Hz}, 1 \mathrm{H}), 7.90(\mathrm{~d}, J=8.2 \mathrm{~Hz}, 1 \mathrm{H}), 8.56$ (s, 1H), 10.43 (br s, NH); Anal. Calcd. for $\mathrm{C}_{15} \mathrm{H}_{10} \mathrm{FNO}_{4} \mathrm{~S}$ (319): C, 56.42; H, 3.16; N, 4.39\%; Found: C, 56.55; H, 3.19; N, 4.36\%.

$\mathbf{N}$-(4-Fluorophenyl)-coumarin-3-sulfonamide (3b). Mp 220-222 ${ }^{\circ} \mathrm{C} ;{ }^{1} \mathrm{H}$ NMR (300 MHz, $\left.\mathrm{CDCl}_{3}+\mathrm{DMSO}_{-} \mathrm{d}_{6}\right): \delta 6.56(\mathrm{~d}, J=7.4 \mathrm{~Hz}, 2 \mathrm{H}), 6.77(\mathrm{~d}, J=7.4 \mathrm{~Hz}, 2 \mathrm{H}), 6.90-7.92(\mathrm{~m}, 4 \mathrm{H}), 8.56$ (s, 1H), 10.42 (br, s, NH); Anal. Calcd. for $\mathrm{C}_{15} \mathrm{H}_{10} \mathrm{FNO}_{4} \mathrm{~S}$ (319): C, 56.42; H, 3.16; N, 4.39\%; Found: C, 56.50; H, 3.31; N, 4.30\%.

$\mathbf{N}$-(3-Chloro-4-fluorophenyl)-coumarin-3-sulfonamide (3c). Mp 188-190 ${ }^{\circ} \mathrm{C} ;{ }^{1} \mathrm{H}$ NMR (300 $\left.\mathrm{MHz}, \mathrm{CDCl}_{3}+\mathrm{DMSO}_{6} \mathrm{~d}_{6}\right): \delta 6.33(\mathrm{~d}, J=7.4 \mathrm{~Hz}, 1 \mathrm{H}), 6.46(\mathrm{~s}, 1 \mathrm{H}), 6.77(\mathrm{~d}, J=7.4 \mathrm{~Hz}, 1 \mathrm{H})$, 6.80-7.88 (m, 4H), $8.56(\mathrm{~s}, 1 \mathrm{H}), 10.42$ (br, s, $1 \mathrm{H})$; Anal. Calcd. for $\mathrm{C}_{15} \mathrm{H}_{9} \mathrm{ClFNO}_{4} \mathrm{~S}(354)$ : C, 50.93; H, 2.56; N, 3.96\%; Found: C, 50.50; H, 2.70; N, 3.78\%.

6-Chloro- $\boldsymbol{N}$-(2-fluorophenyl)-coumarin-3-sulfonamide (3d). Mp 211-212 ${ }^{\circ} \mathrm{C} ;{ }^{1} \mathrm{H}$ NMR (300 $\left.\mathrm{MHz}, \mathrm{CDCl}_{3}\right): \delta$ 6.43-7.77 (m, 6H), $7.80(\mathrm{~s}, 1 \mathrm{H}), 8.60(\mathrm{~s}, 1 \mathrm{H}), 10.42$ (br, s, NH); Anal. Calcd. for $\mathrm{C}_{15} \mathrm{H}_{9} \mathrm{ClFNO}_{4} \mathrm{~S}$ (354): C, 50.93; H, 2.56; N, 3.96\%; Found: C, 50.54; H, 2.51; N, 3.87\%.

6-Chloro- $\boldsymbol{N}$-(4-fluorophenyl)-coumarin-3-sulfonamide (3e). Mp 284-286 ${ }^{\circ} \mathrm{C}$; ${ }^{1} \mathrm{H}$ NMR (300 MHz, DMSO-d 6 ): $\delta$ 6.50-7.98 (m, 8H), $8.60(\mathrm{~s}, 1 \mathrm{H}), 10.42(\mathrm{br}, \mathrm{s}, \mathrm{NH})$; Anal. Calcd. for $\mathrm{C}_{15} \mathrm{H}_{9} \mathrm{ClFNO}_{4} \mathrm{~S}$ (354): C, 50.93; H, 2.56; N, 3.96\%; Found: C, 50.58; H, 2.54; N, 3.82\%.

6-Chloro- $\boldsymbol{N}$-(3-choro-4-fluorophenyl)-coumarin-3-sulfonamide (3f). $\mathrm{Mp} \quad 165-167 \quad{ }^{\circ} \mathrm{C}$; ${ }^{1} \mathrm{H}$ NMR $\left(300 \mathrm{MHz}, \mathrm{CDCl}_{3}\right): \delta 6.35(\mathrm{~d}, J=7.4 \mathrm{~Hz}, 1 \mathrm{H}), 6.44(\mathrm{~s}, 1 \mathrm{H}), 6.76(\mathrm{~d}, J=7.4 \mathrm{~Hz}, 1 \mathrm{H}), 6.77-$ $7.90(\mathrm{~m}, 4 \mathrm{H}), 7.80(\mathrm{~s}, 1 \mathrm{H}), 8.61(\mathrm{~s}, 1 \mathrm{H}), 10.42(\mathrm{br}, \mathrm{s}, 1 \mathrm{H})$; Anal. Calcd. for $\mathrm{C}_{15} \mathrm{H}_{8} \mathrm{Cl}_{2} \mathrm{FNO}_{4} \mathrm{~S}$ (388): C, 46.41; H, 2.08; N, 3.61\%; Found: C, 46.44; H, 2.32; N, 3.66\%.

6-Bromo- $\boldsymbol{N}$-(2-fluorophenyl)-coumarin-3-sulfonamide (3g). Mp 223-225 ${ }^{\circ} \mathrm{C} ;{ }^{1} \mathrm{H}$ NMR (300 $\left.\mathrm{MHz}, \mathrm{CDCl}_{3}\right): \delta 6.43-8.20(\mathrm{~m}, 6 \mathrm{H}), 8.30(\mathrm{~s}, 1 \mathrm{H}), 8.60(\mathrm{~s}, 1 \mathrm{H}), 10.42$ (br, s, NH); Anal. Calcd. for $\mathrm{C}_{15} \mathrm{H}_{9} \mathrm{BrFNO}_{4} \mathrm{~S}$ (398): C, 45.24; H, 2.28; N, 3.52\%; Found: C, 45.34; H, 2.32; N, 3.57\%. 
6-Bromo- $\boldsymbol{N}$-(4-fluorophenyl)-coumarin-3-sulfonamide (3h). Mp 204-206 ${ }^{\circ} \mathrm{C} ;{ }^{1} \mathrm{H}$ NMR (300 MHz, DMSO-d $\left.{ }_{6}\right): \delta 6.50-8.30(\mathrm{~m}, 8 \mathrm{H}), 8.60(\mathrm{~s}, 1 \mathrm{H}), 10.42$ (br, s, NH); C, H, N Anal. Calcd. for $\mathrm{C}_{15} \mathrm{H}_{9} \mathrm{BrFNO}_{4} \mathrm{~S}$ (398): C, 45.24; H, 2.28; N, 3.52\%; Found: C, 45.11; H, 2.26; N, 3.49\%.

6-Bromo- $\boldsymbol{N}$-(2-chloro-4-fluorophenyl)-coumarin-3-sulfonamide (3i). Mp $175-177 \quad{ }^{\circ} \mathrm{C}$; ${ }^{1} \mathrm{H}$ NMR $\left(300 \mathrm{MHz}, \mathrm{CDCl}_{3}\right): \delta 6.41(\mathrm{~d}, J=7.4 \mathrm{~Hz}, 1 \mathrm{H}), 6.42(\mathrm{~s}, 1 \mathrm{H}), 6.77(\mathrm{~d}, J=7.4 \mathrm{~Hz}, 1 \mathrm{H}), 6.76-$ $8.25(\mathrm{~m}, 4 \mathrm{H}), 8.32(\mathrm{~s}, 1 \mathrm{H}), 8.64(\mathrm{~s}, 1 \mathrm{H}), 10.42$ (br, s, $1 \mathrm{H})$; Anal. Calcd. for: $\mathrm{C}_{15} \mathrm{H}_{8} \mathrm{BrClFNO}_{4} \mathrm{~S}$ (433): C, 41.64; H, 1.86; N, 3.24\%; Found: C, 41.78; H, 1.88; N, 3.27\%.

8-Methoxy- $\boldsymbol{N}$-(2-fluorophenyl)-coumarin-3-sulfonamide (3j). Mp 223-225 ${ }^{\circ} \mathrm{C} ;{ }^{1} \mathrm{H}$ NMR (300 $\mathrm{MHz}_{\mathrm{CDCl}}$ ): $\delta 3.93(\mathrm{~s}, 3 \mathrm{H}), 6.43-7.77(\mathrm{~m}, 6 \mathrm{H}), 8.30(\mathrm{~s}, 1 \mathrm{H}), 8.62(\mathrm{~s}, 1 \mathrm{H}), 10.42$ (br, s, NH); Anal. Calcd. for: $\mathrm{C}_{16} \mathrm{H}_{12} \mathrm{FNO}_{5} \mathrm{~S}$ (349): 55.01; H, 3.46; N, 4.01\%; Found: C, 55.11; H, 3.47; N, $4.03 \%$.

8-Methoxy- $\boldsymbol{N}$-(4-fluorophenyl)-coumarin-3-sulfonamide (3k). Mp 181-182 ${ }^{\circ} \mathrm{C} ;{ }^{1} \mathrm{H}$ NMR (300 $\left.\mathrm{MHz}_{\mathrm{CDCl}}\right): \delta 3.93(\mathrm{~s}, 3 \mathrm{H}), 6.30(\mathrm{~d}, J=7.4 \mathrm{~Hz}, 1 \mathrm{H}), 6.46(\mathrm{~s}, 1 \mathrm{H}), 6.80(\mathrm{~d}, J=7.4 \mathrm{~Hz}, 1 \mathrm{H})$, 6.76-7.65 ( m, 4H), $7.14(\mathrm{~s}, 1 \mathrm{H}), 8.63(\mathrm{~s}, 1 \mathrm{H}), 10.42$ (br, s, 1H); Anal. Calcd. for: $\mathrm{C}_{16} \mathrm{H}_{12} \mathrm{FNO}_{5} \mathrm{~S}$ (349): C, 55.01; H, 3.46; N, 4.01\%; Found: C, 55.14; H, 3.48; N, 4.02\%.

8-Methoxy- $\boldsymbol{N}$-(3-chloro-4-fluorophenyl)-coumarin-3-sulfonamide (31). Mp 192-194 ${ }^{\circ} \mathrm{C} ;{ }^{1} \mathrm{H}$ NMR $\left(300 \mathrm{MHz}, \mathrm{CDCl}_{3}\right): \delta 3.93(\mathrm{~s}, 3 \mathrm{H}), 6.34(\mathrm{~d}, J=7.4 \mathrm{~Hz}, 1 \mathrm{H}), 6.46(\mathrm{~s}, 1 \mathrm{H}), 6.66(\mathrm{~d}, J=7.4$ $\mathrm{Hz}, 1 \mathrm{H}), 6.76-7.80$ ( m, 4H), 7.65 (s, 1H), 8.61 (s, 1H), 10.42 (br, s, 1H);

Anal. Calcd. for: $\mathrm{C}_{16} \mathrm{H}_{11} \mathrm{ClFNO}_{5} \mathrm{~S}$ (384): C, 50.07; H, 2.89; N, 3.65\%; Found: C, 55.16; H, 2.88; N, $3.66 \%$.

6-Methyl- $\boldsymbol{N}$-(2-fluorophenyl)-coumarin-3-sulfonamide (3m). Mp 223-225 ${ }^{\circ} \mathrm{C} ;{ }^{1} \mathrm{H}$ NMR (300 MHz, DMSO-d $\left.)_{6}\right): \delta 2.35(\mathrm{~s}, 3 \mathrm{H}), 6.43-7.77(\mathrm{~m}, 6 \mathrm{H}), 7.34(\mathrm{~s}, 1 \mathrm{H}), 8.62(\mathrm{~s}, 1 \mathrm{H}), 10.42(\mathrm{br}, \mathrm{s}, \mathrm{NH})$; Anal. Calcd. for: $\mathrm{C}_{16} \mathrm{H}_{12} \mathrm{FNO}_{4} \mathrm{~S}$ (333): C, 57.65; H, 3.63; N, 4.20\%; Found: C, 57.66; H, 3.64; N, $4.22 \%$.

6-Methyl- $\boldsymbol{N}$-(4-fluorophenyl)-coumarin-3-sulfonamide (3n). Mp 192-194 ${ }^{\circ} \mathrm{C}$; ${ }^{1} \mathrm{H}$ NMR (300 MHz, DMSO-d $\left.)_{6}\right): \delta .36(\mathrm{~s}, 3 \mathrm{H}), 6.34(\mathrm{~d}, J=7.4 \mathrm{~Hz}, 1 \mathrm{H}), 6.44(\mathrm{~s}, 1 \mathrm{H}), 6.66(\mathrm{~d}, J=7.4 \mathrm{~Hz}, 1 \mathrm{H})$, 6.56-7.67 ( m, 4H), $7.65(\mathrm{~s}, 1 \mathrm{H}), 8.62(\mathrm{~s}, 1 \mathrm{H}), 10.42$ (br, s, $1 \mathrm{H})$; Anal. Calcd. for: $\mathrm{C}_{16} \mathrm{H}_{12} \mathrm{FNO}_{4} \mathrm{~S}$ (333): C, 57.65; H, 3.63; N, 4.20\%; Found: C, 57.76; H, 3.66; N, 4.22\%.

6-Methyl- $\boldsymbol{N}$-(3-choro-4-fluorophenyl)-coumarin-3-sulfonamide(3o). Mp $192-194 \quad{ }^{\circ} \mathrm{C} ; \quad{ }^{1} \mathrm{H}$ NMR (300 MHz, DMSO-d 6 ): $\delta 2.35(\mathrm{~s}, 3 \mathrm{H}), 6.44(\mathrm{~d}, J=7.4 \mathrm{~Hz}, 1 \mathrm{H}), 6.47(\mathrm{~s}, 1 \mathrm{H}), 6.64(\mathrm{~d}, J=$ $7.4 \mathrm{~Hz}, 1 \mathrm{H}), 6.76-7.54$ ( m, 4H), 7.55 (s, 1H), 8.61 (s, 1H), 10.42 (br, s, 1H);

Anal. Calcd. for: $\mathrm{C}_{16} \mathrm{H}_{11} \mathrm{ClFNO}_{4} \mathrm{~S}$ (368): C, 52.25; H, 3.01; N, $3.81 \%$; Found: C, 52.16; H, 3.04; $\mathrm{N}, 3.86 \%$.

6-Nitro- $\boldsymbol{N}$-(2-fluorophenyl)-coumarin-3-sulfonamide (3p). $\mathrm{Mp} 223-225{ }^{\circ} \mathrm{C} ;{ }^{1} \mathrm{H}$ NMR (300 MHz, DMSO-d $\left.{ }_{6}\right): \delta 6.43-8.45(\mathrm{~m}, 6 \mathrm{H}), 7.56(\mathrm{~s}, 1 \mathrm{H}), 8.62(\mathrm{~s}, 1 \mathrm{H}), 10.42(\mathrm{br}, \mathrm{s}, \mathrm{NH})$;

Anal. Calcd. for: $\mathrm{C}_{15} \mathrm{H}_{9} \mathrm{FN}_{2} \mathrm{O}_{6} \mathrm{~S}$ (364): C, 49.45; H, 2.49; N, 7.69\%; Found: C, 49.66; H, 2.44; N, $7.66 \%$.

6-Nitro- $\boldsymbol{N}$-(4-fluorophenyl)-coumarin-3-sulfonamide (3q). Mp 192-194 ${ }^{\circ} \mathrm{C} ;{ }^{1} \mathrm{H}$ NMR (300 MHz, DMSO-d $\left.{ }_{6}\right): \delta 6.32(\mathrm{~d}, J=7.4 \mathrm{~Hz}, 1 \mathrm{H}), 6.40(\mathrm{~s}, 1 \mathrm{H}), 6.60(\mathrm{~d}, J=7.4 \mathrm{~Hz}, 1 \mathrm{H}), 6.56-8.40$ 
$(\mathrm{m}, 4 \mathrm{H}), 8.62(\mathrm{~s}, 1 \mathrm{H}), 8.65(\mathrm{~s}, 1 \mathrm{H}), 10.42$ (br, s, $1 \mathrm{H})$; Anal. Calcd. for: $\mathrm{C}_{15} \mathrm{H}_{9} \mathrm{FN}_{2} \mathrm{O}_{6} \mathrm{~S}$ (364): C, 49.45; H, 2.49; N, 7.69\%; Found: C, 49.56; H, 2.56; N, 7.77\%.

6-Nitro- $\boldsymbol{N}$-(3-choro-4-fluorophenyl)-coumarin-3-sulfonamide (3r). Mp 192-194 ${ }^{\circ} \mathrm{C}$; ${ }^{1} \mathrm{H}$ NMR $\left(300 \mathrm{MHz}, \mathrm{DMSO}-\mathrm{d}_{6}\right): \delta 6.44(\mathrm{~d}, J=7.4 \mathrm{~Hz}, 1 \mathrm{H}), 6.47(\mathrm{~s}, 1 \mathrm{H}), 6.64(\mathrm{~d}, J=7.4 \mathrm{~Hz}, 1 \mathrm{H}), 6.76-$ $8.34(\mathrm{~m}, 4 \mathrm{H}), 8.62(\mathrm{~s}, 1 \mathrm{H}), 8.64(\mathrm{~s}, 1 \mathrm{H}), 10.42$ (br, s, $1 \mathrm{H})$; Anal. Calcd. for: $\mathrm{C}_{15} \mathrm{H}_{8} \mathrm{ClFN}_{2} \mathrm{O}_{6} \mathrm{~S}$ (399): C, 45.18; H, 2.02; N, 7.03\%; Found: C, 44.29; H, 2.14; N, 7.15\%.

\section{Acknowledgements}

The work was supported by SMBA-Korea (2007-S1016568).

\section{References}

1. (a) Fylaktakidou, K. C.; Hadjipavlou-Litina, D. J.; Litinas, K. E.; Nicolaides, D. N. Curr. Pharm. Design 2004, 10, 3813. (b) Xie, L.; Takeuchi, Y.; Cosentino, L. M.; McPhail, A. T.; Lee, K. H. J. Med. Chem. 2001, 44, 664. (c) Yang, Z. Y.; Xia, Y.; Xia, P.; Brossi, A.; Cosentino, L. M.; Lee, K.-H. Bioorg. Med. Chem. Lett. 2000, 10, 1003.

2. Pechmann, H.; Duisberg, C. Chem. Ber. 1883, 16, 2119.

3. Frèere, S.; Thiéry V.; Besson T. Tetrahedron Lett. 2001, 42, 2791.

4. Singh, V.; Kaur, S.; Sapehiyia, V.; Singh. J.; Kad, G. L. Catal. Commun. 2005, 6, 57.

5. Palaniappan, S.; Shekhar, R. C. J. Mol. Catal. A: Chemical 2004, 209, 117.

6. Laufer, M. C.; Hausmann, H.; Hölderich, W. F. J. Catal. 2003, $218,315$.

7. Kadnikov, D. V.; Larock, R. C. J. Organomet. Chem. 2003, 687, 425.

8. Jones, G. Organic Reactions; John Wiley \& Sons: New York, 1967; Vol. 15, pp 204-599.

9. Bigi, F.; Chesini, L.; Maggi, R.; Sartori, G. J. Org. Chem. 1999, 64, 1033.

10. Hepworth, J. D. Comprehensive Heterocyclic Chemistry; Pergamon Press: Oxford, 1984; Vol. 3, pp 799-809.

11. Ishii, H.; Ishikawa, T.; Wada, H.; Miyazaki, H.; Kanenko, Y.; Harayama, T. Chem. Pharm. Bull. 1992, 40, 2614.

12. Pimentel, L. C. F.; de Souza, A. L. F.; Fernández, T. L.; Wardell, J. L.; Antunes, O. A. C. Tetrahedron Lett. 2007, 48, 831.

13. Zoni, F.; Vicini, P. Arch. Pharm. 1998, 331, 219.

14. Li, J. J., Anderson, D.; Burton, E. G.; Cogburn, J. N.; Collins, J. T.; Garland, D. J.; Gregory, S. A.; Huang, H. C.; Isakson, P. C.; Koboldt, C. M.; Logusch, E. W.; Norton, M. B.; Perkins, W. E.; Reinhard, E. J.; Seibert, K.; Veenhuizem, A. W.; Zang, Y.; Reitz, D. B. J. Med. Chem. 1995, 38, 4570.

15. Yoshino, H.; Ueda, N.; Niijima, J.; Sugumi, H.; Kotake, Y.; Koyanagi, N.; Yoshimatsu, K.; Asada, M.; Watanabe, T.; Nagasu, T.; Tsukahara, K.; Lijima, A.; Kitoh, K. J. Med. Chem. 1992, 35, 2496. 